\title{
Measurement of the magnetic material properties for ferrite-loaded cavities
}

\author{
Klaus Klopfer, ${ }^{1, *}$ Uwe Niedermayer, ${ }^{1}$ Harald Klingbeil, ${ }^{1,2}$ Wolfgang Ackermann, ${ }^{1}$ \\ Hans Günter König, ${ }^{2}$ and Thomas Weiland ${ }^{1}$ \\ ${ }^{1}$ Technische Universität Darmstadt, Institut für Theorie Elektromagnetischer Felder (TEMF), \\ Schlossgartenstraße 8, 64289 Darmstadt, Germany \\ ${ }^{2}$ GSI Helmholtzzentrum für Schwerionenforschung GmbH, Planckstr. 1, 64291 Darmstadt, Germany
}

(Received 3 June 2014; published 30 January 2015)

\begin{abstract}
Measurements of the magnetic characteristics of the Ferroxcube $8 \mathrm{C} 12 \mathrm{~m}$ ferrite material in the parameter range where the GSI heavy-ion synchrotron SIS 18 cavity resonator is operated are presented. At first, the permeability is determined as a function of frequency and bias magnetic field strength for low radiofrequency power levels. For this purpose, both reflection and transmission measurements are carried out in a test setup with two toroids. The values for the real and imaginary part obtained from the data analysis of both approaches are fully in agreement with each other, albeit the range of application of the latter setup is limited to moderate frequencies due to parasitic resonances. An empirical analytical expression is formulated which approximates the complex permeability reasonably well in the whole investigated bias and frequency range. Moreover, the $B-H$ curve is recorded for a reduced bias current range of the cavity. The gained material characteristics are well suited for numerical eigenmode simulations for the GSI SIS 18 cavity.
\end{abstract}

DOI: 10.1103/PhysRevSTAB.18.010101

PACS numbers: 75.50.-y, 85.70.Ge, 29.20.dk

\section{INTRODUCTION}

Ferrite-loaded cavity resonators are widely used for the acceleration of heavy ions in ramped operation in synchrotrons for two main reasons: On the one hand, the high permeability of the ferrite material causes a reduced wavelength compared to vacuum. On the other hand, the material characteristics of ferrites depend on the external bias magnetic field. This is employed for tuning of the resonance frequency of the resonator according to the increasing revolution frequency of the heavy ions.

The new international Facility for Antiproton and Ion Research (FAIR) will be built at the GSI Helmholtzzentrum für Schwerionenforschung in Darmstadt in the next years. One of the main components will be the double ring synchrotron SIS 100/300 with a circumference of $1.1 \mathrm{~km}$, for which the already existing GSI heavy-ion synchrotron SIS 18 will serve as an injector. Within the SIS 18 two cavities are operated, which are loaded with the Ferroxcube $8 \mathrm{C} 12 \mathrm{~m}$ ferrite material. It is foreseen that several accelerating cavities of the same type will be installed in the SIS 100 synchrotron with slight modifications. For that reason, it is the final aim of the presented study to develop a tool for the numerical simulation of the lowest eigenmodes of such resonators. This may also provide means for a better understanding of the tuning process. Evidently, accurate material characteristics are required for the numerical

\footnotetext{
*klopfer@temf.tu-darmstadt.de

Published by the American Physical Society under the terms of the Creative Commons Attribution 3.0 License. Further distribution of this work must maintain attribution to the author(s) and the published article's title, journal citation, and DOI.
}

computations. Among the most important properties are the dependence of the permeability on the frequency and the bias magnetic field strength. Since only limited information is available in the data sheet provided by the manufacturer, goal-directed measurements were carried out at the GSI facility.

This paper is structured as follows. After elaborating on the setup for the determination of the magnetic characteristics of the ferrite, the analysis of the reflection and transmission measurement data is discussed. Subsequently, the measurement results for the $B-H$ curve, which is enclosed in a reduced working cycle of the cavity, are presented.

\section{PERMEABILITY MEASUREMENT}

\section{A. Setup}

Two different methods are used for the measurement of the frequency dependent characteristics. Whereas the first approach is based on the measurement of the input port reflection coefficient, the $S_{11}$-parameter, transmission measurements of $S$ parameters are carried out in the second one. The underlying principle of the two methods is similar to approaches known from literature, namely, for instance [1-3] for the reflection measurement and [4-6] for the transmission measurement. The basic setting of the elaborated setup of both approaches is identical: Two Ferroxcube $8 \mathrm{C} 12 \mathrm{~m}$ full size toroids of outer radius $r_{\text {out }}=$ $249 \mathrm{~mm}$ and height $h_{\text {core }}=50 \mathrm{~mm}$ can be biased via $N_{\text {bias }}=105$ crossed (figure-of-eight) current windings. The bias current is adjusted manually at a direct current (dc) power supply (Delta Elektronika SM 45-70D [7]). Moreover, the rings together with the current windings are installed inside a copper cavity housing. The outer surface is not fully closed (cf. Fig. 1). Unlike as in the GSI SIS 18 


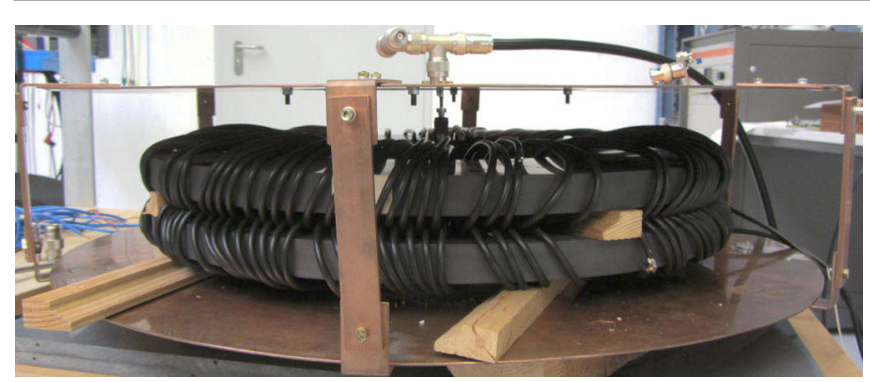

FIG. 1. Experimental setup in the reflection measurement mode. The ferrite ring pair with bias windings is located inside the cavity housing. The outer radius of each ring core is $r_{\text {out }}=249 \mathrm{~mm}$; its height is $h_{\text {core }}=50 \mathrm{~mm}$. Further dimensions of the setup are given in Table II and Fig. 3. Note that the housing is not fully closed at the outer surface. The $\mathrm{N}$-type cable in the center on top is connected to the VNA. A $50 \Omega$ resistor can be installed in parallel.

cavity, no cooling disk is installed between the two toroids. For the first approach, this housing together with one centric wire, which connects the upper and lower plate, also serves for the coupling of the radio frequency (rf) to the ring cores. In contrast, for the second approach the inner wire is disconnected. Instead, two separate current windings wound closely around both ring cores are installed for excitation and pickup of the signal. The $S$ parameters of the device under test are measured with a Rohde and Schwarz ZNB4 vector network analyzer (VNA) [8] in the frequency range from 0.5 to $6 \mathrm{MHz}$. The used settings of the network analyzer are listed in Table I. Figure 1 shows the measurement station for the reflection setup.

In order to prepare the magnetic remanence state, the bias current is driven up to the maximum value of $50 \mathrm{~A}$ $(H \approx 4.6 \mathrm{kA} / \mathrm{m})$ for a short time in an initialization step. The bias current was not further increased to avoid excessive heating of the ferrite. Yet, the provided magnetic field strength is much larger than the available value for the magnetic field strength to reach the saturated state $(0.8 \mathrm{kA} / \mathrm{m})$ given in the supplier's data handbook for the unmodified Ferroxcube 8C12 material [9]. Moreover, since the measurements could be well reproduced after having performed the initialization starting from a previously undefined magnetic state, it can be assumed that a magnetically sufficiently saturated state is reached.

The measurement is performed for values of the bias current starting with $0 \mathrm{~A}$ and raising up to the maximum value with enlarging increments at room temperature.

TABLE I. Common settings of the network analyzer for both measurement methods.

\begin{tabular}{lc}
\hline \hline Number of sample points & 4001 \\
Sweep type & logarithmic frequency \\
Source power & $0 \mathrm{dBm}$ \\
Frequency range & $0.5 \mathrm{MHz}$ to $6 \mathrm{MHz}$ \\
Bandwidth & $1 \mathrm{kHz}$ \\
Averaging & 4 times \\
\hline \hline
\end{tabular}

Moreover, a careful calibration including the N-type cables was performed initially. For the transmission measurement, it included a full one-port calibration with an open, short and match standard as for the reflection measurement for each port as well as a standard through connection. A high phase stability was observed.

\section{B. Analysis of the reflection measurement data}

\section{General procedure}

After the reflection measurement, the $S_{11}$-parameter is available as a function of the frequency for the different bias current and capacitor settings, which are transformed to the real and imaginary part of the admittance $Y$. Assuming that the system can be described by a circuit as depicted in Fig. 2, its total admittance is given by

$$
Y=\frac{1}{R_{0}}+\mathrm{i} \omega C_{\mathrm{dist}}+\frac{1}{\mathrm{i} \omega L_{\mathrm{s}}+R_{\mathrm{s}}}
$$

with the distributed capacitance $C_{\text {dist }}$, the parallel resistor $R_{0}=50 \Omega$ and the series inductance $L_{\mathrm{s}}$ and resistance $R_{\mathrm{s}}$ of the two toroids. In reality, the parallel resistor is not an ideal component. To account for this, an additional measurement is carried out to obtain the admittance

$$
Y_{0}=\frac{1}{R_{0}}
$$

of this component alone without being connected to the device under test. Hence, the inductance $L_{\mathrm{s}}$ and the resistance $R_{\mathrm{S}}$ of the ring cores can be expressed in terms of $Y$ and $Y_{0}$ as

$L_{\mathrm{s}}=\frac{\omega C_{\mathrm{dist}}-\operatorname{Im}(Y)+\operatorname{Im}\left(Y_{0}\right)}{\omega\left\{\left[\operatorname{Re}(Y)-\operatorname{Re}\left(Y_{0}\right)\right]^{2}+\left[\operatorname{Im}(Y)-\operatorname{Im}\left(Y_{0}\right)-\omega C_{\mathrm{dist}}\right]^{2}\right\}}$

and $R_{\mathrm{s}}=\frac{\operatorname{Re}(Y)-\operatorname{Re}\left(Y_{0}\right)}{\left[\operatorname{Re}(Y)-\operatorname{Re}\left(Y_{0}\right)\right]^{2}+\left[\operatorname{Im}(Y)-\operatorname{Im}\left(Y_{0}\right)-\omega C_{\text {dist }}\right]^{2}}$,

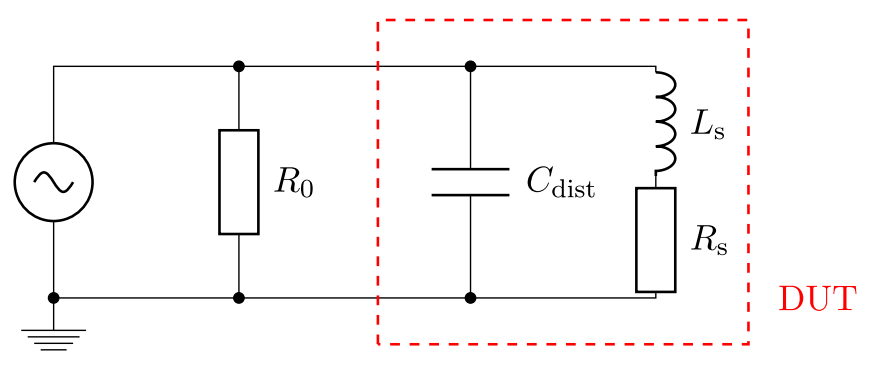

FIG. 2. Equivalent circuit diagram to represent the measurement setup. The resistor $R_{0}$ is installed in parallel to the device under test (DUT). The DUT itself is modeled as a distributed capacitance $C_{\text {dist }}$ in parallel with a series inductance $L_{\mathrm{s}}$ and resistance $R_{\mathrm{s}}$. 


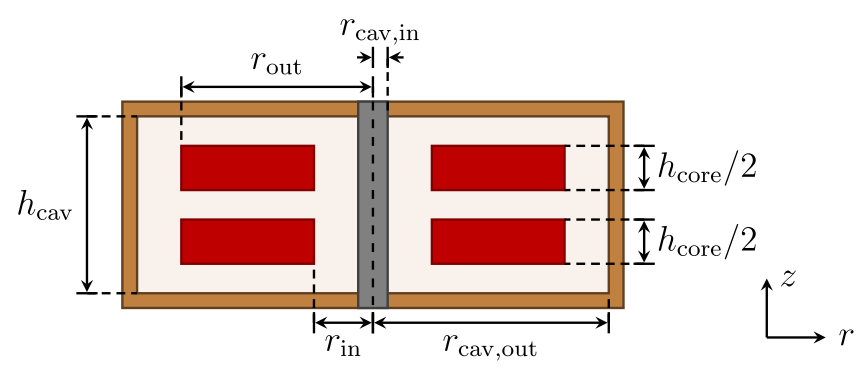

FIG. 3. Simplified two-dimensional sectional view through the center of the cavity. The dimensions of the cavity and the ring cores are given in Table II.

respectively. Moreover, these quantities are related to the permeability $\mu_{\mathrm{s}}=\mu_{\mathrm{s}}^{\prime}-\mathrm{i} \mu_{\mathrm{s}}^{\prime \prime}$ involving the dimensional parameters as depicted in Fig. 3 by the relations [3]

$$
\begin{aligned}
& L_{\mathrm{s}}=\frac{1}{\underline{I}} \int \underline{B} \mathrm{~d} A=\int_{r_{\text {cav }, \text { in }}}^{r_{\text {cav out }}} \int_{0}^{h_{\mathrm{cav}}} \frac{\mu_{\mathrm{s}}^{\prime}}{2 \pi r} \mathrm{~d} r \mathrm{~d} z, \\
& R_{\mathrm{s}}=\frac{\mathrm{i} \omega}{\underline{I}} \int \underline{B} \mathrm{~d} A=\omega \int_{r_{\text {cav }, \text { in }}}^{r_{\text {cav }, \text { out }}} \int_{0}^{h_{\mathrm{cav}}} \frac{\mu_{\mathrm{s}}^{\prime \prime}}{2 \pi r} \mathrm{~d} r \mathrm{~d} z .
\end{aligned}
$$

This can be transformed into

$$
\begin{aligned}
& L_{\mathrm{s}}=\frac{1}{2 \pi} h_{\text {core }}\left(\mu_{\mathrm{s}}^{\prime}-\mu_{0}\right) \ln \frac{r_{\text {out }}}{r_{\text {in }}}+L_{\text {air }}, \\
& R_{\mathrm{s}}=\frac{\omega}{2 \pi} h_{\text {core }} \mu_{\mathrm{s}}^{\prime \prime} \ln \frac{r_{\text {out }}}{r_{\text {in }}}+R_{\text {cav }}
\end{aligned}
$$

with the inductance of the air-filled resonator

$$
L_{\text {air }}=\frac{1}{2 \pi} h_{\mathrm{cav}} \mu_{0} \ln \frac{r_{\mathrm{cav}, \mathrm{out}}}{r_{\mathrm{cav}, \mathrm{in}}}
$$

The resistance of the empty cavity $R_{\text {cav }}$ is yet to be determined. From Eqs. (5), the real and imaginary part of the permeability are finally obtained as

TABLE II. Dimensions of the cavity and the ring cores. The quantities are illustrated in Fig. 3.

\begin{tabular}{lc}
\hline \hline Quantity & Value \\
\hline$h_{\text {core }}$ & $50 \mathrm{~mm}$ \\
$r_{\text {out }}$ & $249 \mathrm{~mm}$ \\
$r_{\text {in }}$ & $136 \mathrm{~mm}$ \\
$r_{\text {cav,out }}$ & $340 \mathrm{~mm}$ \\
$r_{\text {cav,in }}$ & $1.75 \mathrm{~mm}$ \\
$h_{\text {cav }}$ & $143 \mathrm{~mm}$ \\
\hline \hline
\end{tabular}

$$
\begin{aligned}
& \mu_{\mathrm{s}}^{\prime}=\left(L_{\mathrm{s}}-L_{\text {air }}\right) \frac{2 \pi}{h_{\text {core }} \ln \left(r_{\text {out }} / r_{\text {in }}\right)}+\mu_{0}, \\
& \mu_{\mathrm{s}}^{\prime \prime}=\left(R_{\mathrm{s}}-R_{\text {cav }}\right) \frac{2 \pi}{\omega h_{\text {core }} \ln \left(r_{\text {out }} / r_{\text {in }}\right)}
\end{aligned}
$$

with $L_{\mathrm{s}}$ and $R_{\mathrm{s}}$ as given in Eqs. (3). Equation (6) allows only a rather inaccurate determination of the inductance of the empty cavity in practice. Hence, the inductance $L_{\text {air }}$ and also the resistance $R_{\text {cav }}$ of the empty cavity are obtained from a separate measurement without the toroids as explained in Sec. II B 2. The distributed capacitance depends only weakly on frequency in good approximation [1]. From practical considerations, a value of $C_{\text {dist }}=10 \mathrm{pF}$ is assumed. This value is in accordance with numerical eigenvalue simulations of the cavity resonator loaded with two ring cores of a material with a spatially constant as well as frequency-independent permeability and a permittivity in the range as stated in the data sheet for the Ferroxcube material [9]. Note also that the finally obtained values for the permeability are rather insensitive to $C_{\text {dist }}$ for frequencies up to $6 \mathrm{MHz}$, which justifies one to assume a frequency-independent value of the distributed capacitance for the analysis.

Moreover, the applied magnetic bias field $H_{\text {bias }}$ can be expressed as [9]

$$
H_{\text {bias }}=\frac{N_{\text {bias }} I}{l_{\text {eff }}}
$$

with the effective magnetic path length $l_{\text {eff }}$. For simple toroidal shapes $l_{\mathrm{eff}}$ is calculated as [10]

$$
l_{\text {eff }}=\frac{2 \pi \ln \left(r_{\text {out }} / r_{\text {in }}\right)}{1 / r_{\text {in }}-1 / r_{\text {out }}} .
$$

\section{Measurement of the inductance $L_{\text {air }}$ and the resistance $R_{\text {cav }}$ of the empty cavity}

In order to determine the inductance $L_{\text {air }}$ and the resistance $R_{\text {cav }}$ from measurement, the admittance $Y_{\text {air }}$ of the empty cavity without the ferrite ring cores is recorded. Its real and imaginary part are then fitted to the function

$$
Y_{\mathrm{air}}=\frac{1}{R_{0}}+\frac{1}{\mathrm{i} \omega L_{\mathrm{air}}+R_{\mathrm{cav}}}
$$

keeping $L_{\text {air }}$ and $R_{\text {cav }}$ as free parameters. As is visible in Fig. 4, it is possible to reproduce $Y_{\text {air }}$ very well with frequency-independent values of $L_{\text {air }}=180 \pm 8 \mathrm{nH}$ and $R_{\text {cav }}=17.5 \pm 17.5 \mathrm{~m} \Omega$. The stated uncertainty estimations take into account the accuracy of the VNA as given by the manufacturer [11].

As a cross-check, the value of $L_{\text {air }}$ is also calculated according to Eq. (6), its uncertainty according to quadratic error propagation with the estimated uncertainties 

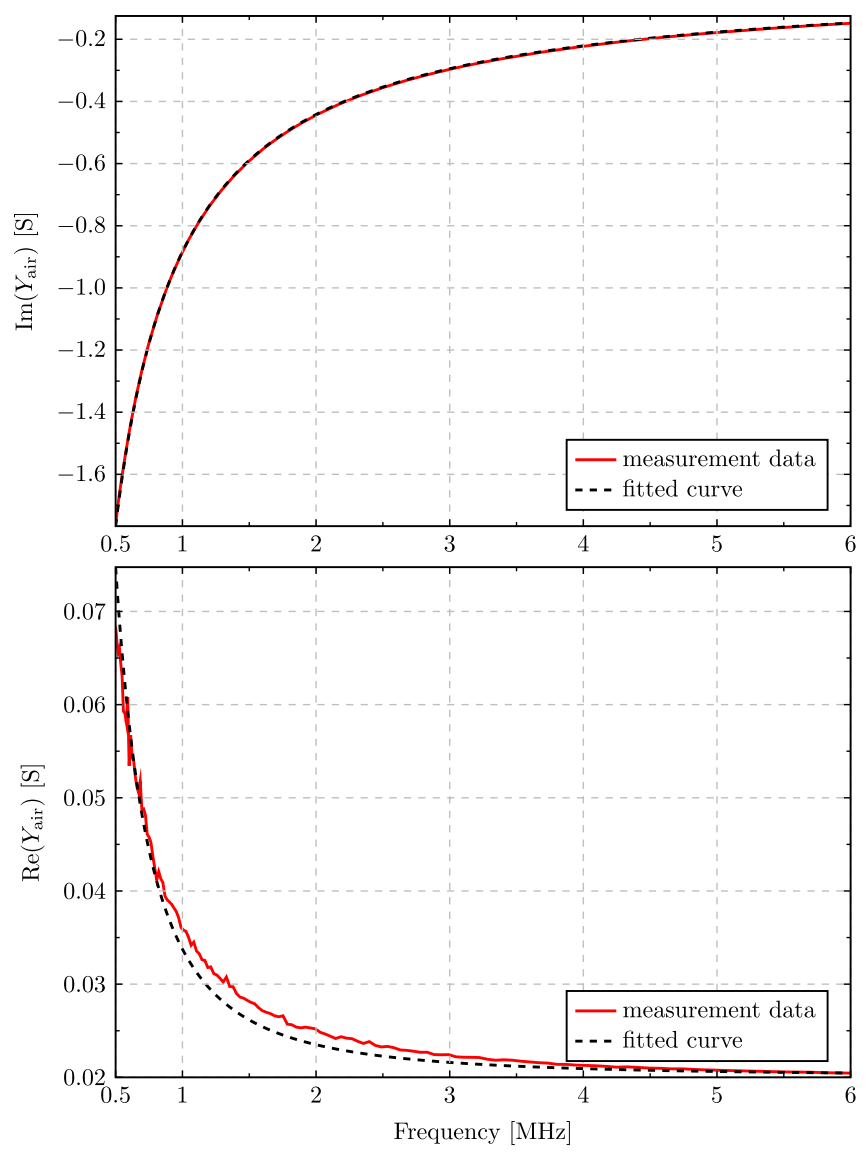

FIG. 4. Imaginary part (above) and real part (below) of the admittance $Y_{\text {air }}$ of the empty cavity as a function of frequency for the reflection measurement. The fitted curve (black dashed curve) is shown with $L_{\text {air }}=180 \mathrm{nH}$ and $R_{\text {cav }}=17.5 \mathrm{~m} \Omega$ as obtained by a fit to Eq. (10).

$\Delta h_{\text {cav }}=3 \mathrm{~mm}, \Delta r_{\text {cav }, \text { out }}=5 \mathrm{~mm}$, and $\Delta r_{\text {cav, in }}=0.2 \mathrm{~mm}$ for the input quantities. This way a value of $L_{\text {air }}=151 \pm$ $7 \mathrm{nH}$ is found, which is not fully in accordance with the measured value. Possible reasons for the slight deviation are additional inductive contributions such as those from the inner wire and the cavity housing, which are not included in the simple calculation. Moreover, an estimation of the resistance $R_{\text {cav }}$ from an analytical calculation taking into account the skin depth of the copper material leads to a value compatible with the measured one.

\section{Uncertainty analysis}

To estimate the accuracy of the obtained measurement results, an extensive error analysis is performed, which takes into account the uncertainty of all input parameters. The analysis only investigates random errors whereas possible systematic errors are not taken into account. It reveals that the accuracy of the VNA has a major impact on the uncertainty of the permeability. Though the uncertainty of the distributed capacitance plays an increasingly important role with raising frequency, it is of minor importance in the analyzed frequency range. In general, the lowest relative error is observed for a low bias magnetic field strength up to moderate frequencies.

\section{Analysis of the transmission measurement data}

Within the transmission measurement, the full twoport scattering matrix is recorded. The complex permeability is then extracted as derived in the following. The corresponding circuit diagram is shown in Fig. 5.

The variable-frequency harmonic oscillator of the network analyzer drives a current $\underline{I}_{1}(t)=\underline{\underline{I}}_{1} \exp (\mathrm{i} \omega t)$ through the primary winding with $N_{1}$ primary turns, which in turn excites the (complex) magnetic field strength

$$
\underline{H}(t, r)=N_{1} \frac{\underline{I}_{1}(t)}{2 \pi r}
$$

inside the toroids [4]. The secondary winding with $\mathrm{N}_{2}$ turns, which is used as sense winding, comprises the magnetic flux (cf. Table III and Fig. 6)

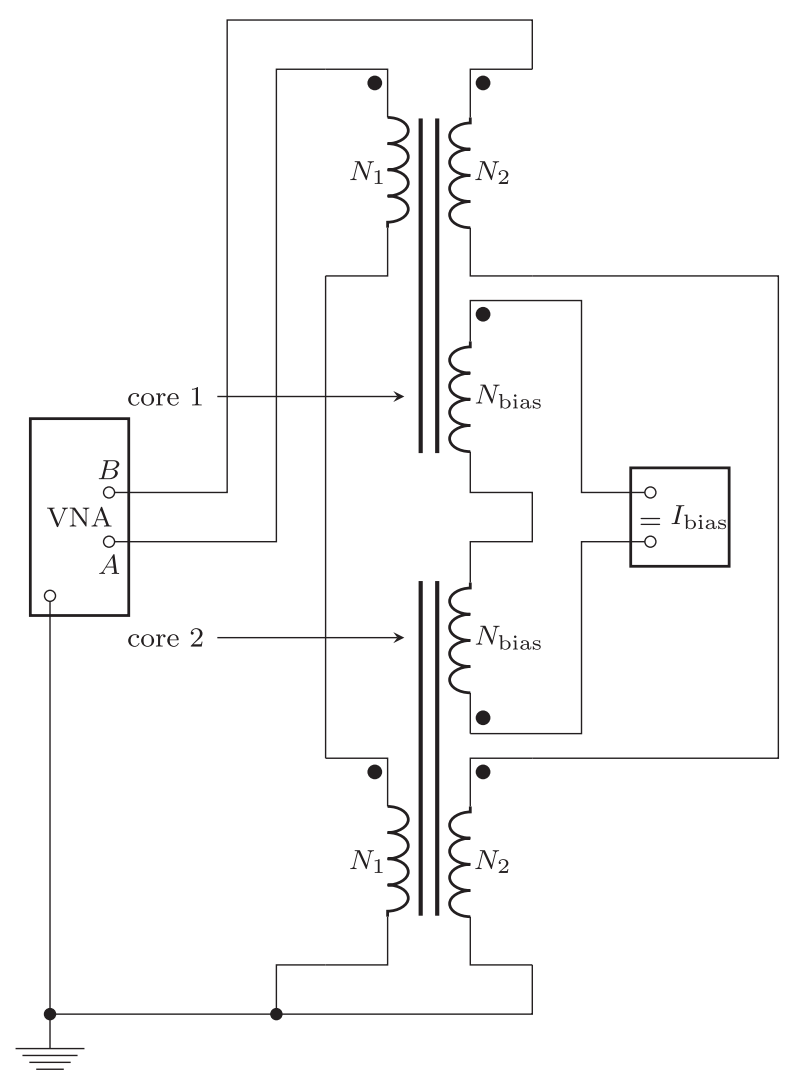

FIG. 5. Circuit diagram for the transmission measurement. The primary winding with $N_{1}$ turns and the secondary winding with $N_{2}$ turns are connected to ports $A$ and $B$ of the VNA, respectively. The cores under test are biased via $N_{\text {bias }}=105$ crossed (figure-of-eight) current windings. 


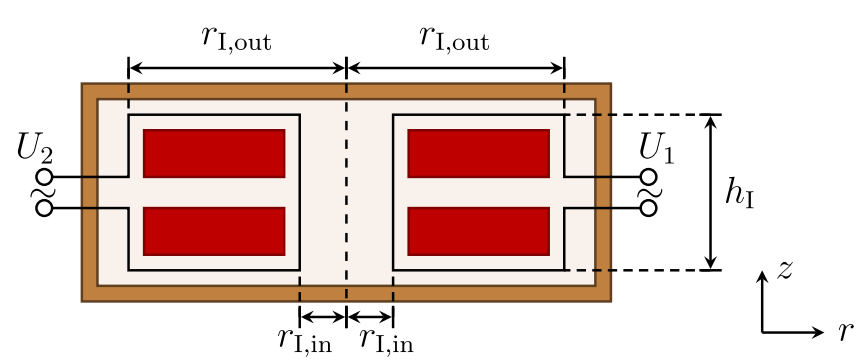

FIG. 6. Simplified two-dimensional sectional view through the center of the cavity to illustrate geometric quantities related to the location of the primary and secondary winding. The values of the quantities are given in Table III.

$$
\begin{aligned}
\underline{\Phi}(t) & =\int_{r_{\mathrm{I}, \text { out }}} \underline{\vec{B}}(t) \cdot \mathrm{d} \vec{A} \\
& =\int_{r_{\mathrm{I} \text {,in }}}\left[\left(\underline{\mu}_{\text {core }}-\mu_{0}\right) h_{\text {core }}+\mu_{0} h_{\mathrm{I}}\right] \underline{H}(t, r) \mathrm{d} r \\
& =\frac{N_{1} \underline{I}_{1}(t)}{2 \pi}\left(\underline{\mu}_{\text {core }} h_{\text {core }} \ln \frac{r_{\text {out }}}{r_{\text {in }}}+\Psi_{\text {air }}\right)
\end{aligned}
$$

with $\Psi_{\text {air }}:=-\mu_{0} h_{\text {core }} \ln \frac{r_{\text {out }}}{r_{\text {in }}}+\mu_{0}\left(h_{\text {core }}+h_{\mathrm{I}}\right) \ln \frac{r_{\mathrm{I}, \text { out }}}{r_{\mathrm{I}, \text { in }}}$,

where $\underline{\mu}_{\text {core }}$ is the complex permeability of the ferrite ring cores. Note that the global sign on the right-hand side of Eq. (12a) depends on the relative orientation of excitation and sense winding. By Faraday's law the voltage

$$
\begin{aligned}
\underline{U}_{2}(t) & =\underline{U}_{2} \exp (\mathrm{i} \omega t) \\
& =-N_{2} \frac{\mathrm{d} \underline{\Phi}(t)}{\mathrm{d} t} \\
& =-\mathrm{i} \omega \frac{N_{1} N_{2} \underline{I}_{1}(t)}{2 \pi}\left(\underline{\mu}_{\text {core }} h_{\text {core }} \ln \frac{r_{\text {out }}}{r_{\text {in }}}+\Psi_{\text {air }}\right)
\end{aligned}
$$

is induced in the sense winding. Solving this complexvalued equation for the real and imaginary part of the permeability in the series representation, i.e. $\underline{\mu}_{\text {core }}:=\mu_{\mathrm{s}}^{\prime}-\mathrm{i} \mu_{\mathrm{s}}^{\prime \prime}$, finally yields

$$
\mu_{\mathrm{s}}^{\prime}=-\frac{1}{h_{\text {core }} \ln \left(r_{\text {out }} / r_{\text {in }}\right)}\left(\frac{2 \pi \operatorname{Im}\left(Z_{21}\right)}{N_{1} N_{2} \omega}+\Psi_{\text {air }}\right),
$$

TABLE III. Geometric quantities related to the location of the primary and secondary winding. The quantities are illustrated in Fig. 6.

\begin{tabular}{lc}
\hline \hline Quantity & Value \\
\hline$h_{\mathrm{I}}$ & $71 \mathrm{~mm}$ \\
$r_{\mathrm{I} \text { out }}$ & $254 \mathrm{~mm}$ \\
$r_{\mathrm{I} \text {,in }}$ & $131 \mathrm{~mm}$ \\
\hline \hline
\end{tabular}

$$
\mu_{\mathrm{s}}^{\prime \prime}=-\frac{1}{h_{\text {core }} \ln \left(r_{\text {out }} / r_{\text {in }}\right)} \times \frac{2 \pi \operatorname{Re}\left(Z_{21}\right)}{N_{1} N_{2} \omega}
$$

involving the impedance parameter [12]

$\underline{Z}_{21}:=\left.\frac{\hat{U}_{2}}{\underline{\hat{I}}_{1}}\right|_{I_{2}=0}=\frac{2 S_{21}}{\left(1-S_{11}\right)\left(1-S_{22}\right)-S_{12} S_{21}} Z_{0}$,

where $Z_{0}=50 \Omega$ is the characteristic impedance.

\section{Measurement results}

The measurement results for the permeability are presented in the following. In Sec. II D 1 the obtained values for the complex permeability as a function of frequency and bias magnetic field strength are discussed including a comparison of the results for the reflection and transmission method. Additionally, an empirical analytical expression is stated and motivated from theoretical considerations. Subsequently, the reversible permeability is shown as a function of bias magnetic field strength in Sec. II D 2. Finally, the product of $\mu_{\mathrm{s}}^{\prime}$, the quality factor $Q$, and the frequency, the $\mu Q f$ product, which serves as a figure of merit for the ferrite, is presented in Sec. II D 3. Note that except for the comparison in Sec. II D 1, only the obtained values from the reflection method are used throughout this work for the evaluation of derived quantities.

\section{Permeability as a function of frequency and bias magnetic field strength}

Reflection measurement.-The values obtained from the analysis of the reflection measurement data for the real and imaginary parts of the permeability in the series representation as a function of frequency for a few selected bias magnetic field strengths are shown in Fig. 7. In the original measurement data, distortions in the real part of the admittance at low frequencies are observed, which result in sharp resonances, where the value of $\mu_{\mathrm{s}}^{\prime \prime}$ is increased (cf. Fig. 7). These distortions are present only for a nonvanishing bias current between $\approx 0.5$ and $\approx 2.5 \mathrm{MHz}$. Furthermore, their amplitude first increases with bias current, reaches a maximum at around $I=3 \mathrm{~A}$ $\left(H_{\text {bias }} \approx 0.3 \times 10^{3} \mathrm{~A} / \mathrm{m}\right)$ and then gradually decreases. At the same time, the distortions move to higher frequencies. It is speculated that they are caused by undesired interactions between the dc current generator and distributed capacitances of the bias current windings. This suspicion is substantiated by the observation that the frequency of the distortions changes when the bias windings are additionally grounded somewhere in between. Exploiting this, these distortions could eventually be eliminated by combining different sets of data which were taken with and without additional grounding. Furthermore, as will be seen in the next paragraph, the same distortions also occur for the transmission measurement, for which, however, no correction has been performed. 


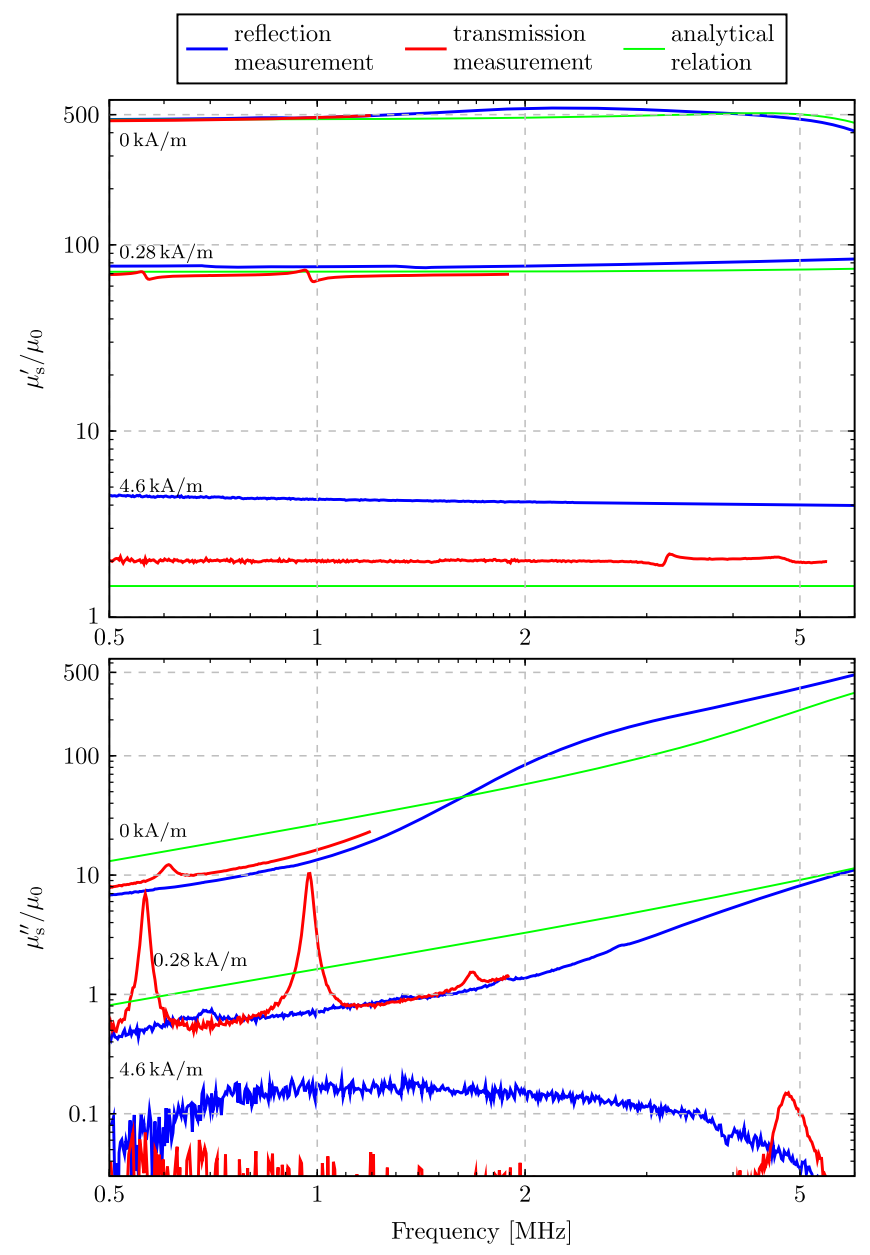

FIG. 7. Comparison of the real part (above) and the imaginary part (below) of the permeability $\mu_{\mathrm{s}}$ as obtained from the reflection and transmission measurement analysis for three different bias magnetic field strengths. Unlike in the analysis for the reflection setup, no corrections for the distortions, which result in sharp resonances, are applied for the transmission measurement. The empirically found analytical relation (18) is also plotted for comparison.

Moreover, Fig. 8 demonstrates the dependence of $\mu_{\mathrm{s}}^{\prime}$ and $\mu_{\mathrm{s}}^{\prime \prime}$ on the bias magnetic field strength as a function of frequency. The relative permeability of about 4 for the real part at the maximal bias field $4.6 \mathrm{kA} / \mathrm{m}$ might suggest that the magnetically fully saturated state is not yet reached. Yet, it should be taken into account that the relative uncertainty of the obtained permeability values increases with raising bias field strength. In the region of maximal bias field, the estimated uncertainty is in the same order as the value itself. This is also suggested by the observation that the independent transmission measurement yields approximately only half the value of the reflection measurement for the real part of the permeability at $4.6 \mathrm{kA} / \mathrm{m}$ (cf. Fig. 7) although very small relative deviations of the two approaches are observed for low magnetic bias.

Generally, the results could be well reproduced after dissembling and restoring the whole setup. In order to

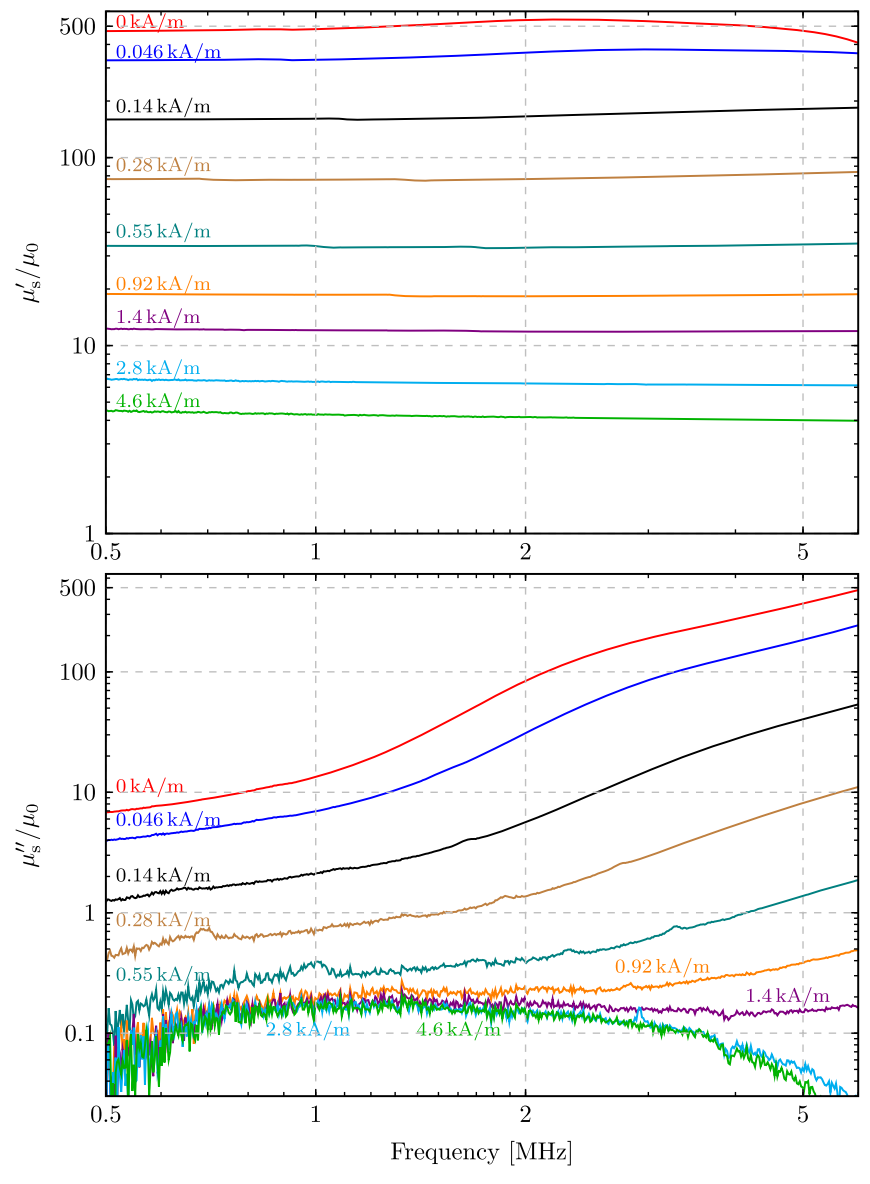

FIG. 8. Real part (above) and imaginary part (below) of the permeability $\mu_{\mathrm{s}}$ as obtained from the reflection measurement analysis with different bias field strengths as a function of frequency.

check the sensitivity on the rf level, tests with different settings for the source of the VNA were carried out for the reflection measurements. No significant deviations were observed for a source power between -20 and $10 \mathrm{dBm}$ except for the increasing noise at very low power levels. Transmission measurement.-The analysis of the transmission parameter $\underline{Z}_{21}$ reveals that the measured data are affected by undesired resonances. Experiments demonstrated that the resonance frequency decreases by a factor of approximately $\sqrt{2}$ when doubling the number of sense windings but is rather insensitive with regard to the selfinductance of the windings. Such $L C$ resonances are known from literature [4]. In the elaborated setup, the resonance frequency is around $8 \mathrm{MHz}$ without biasing. It increases with raising bias current since the resulting real part of the permeability of the ferrites is decreased. It thus leads to a reduced inductance and finally increased resonance frequency. Nevertheless, an evaluation of the data sufficiently far below the resonance is attempted in the following. To allow an easier comparison of the results from the reflection with the transmission measurement, the obtained values for $\mu_{\mathrm{s}}^{\prime}$ and $\mu_{\mathrm{s}}^{\prime \prime}$ are plotted in Fig. 7 for three selected bias field 
strengths. As can be seen there, the values are fully in accordance with the ones of the reflection measurement.

The evaluated data presented so far were recorded in a setting where the sense and the primary winding were placed diametrically opposed on the ring cores. In order to check the influence of their relative position, the transmission measurements were repeated with another sense winding which was installed at an angle of $90^{\circ}$ with respect to the primary winding. The obtained $\underline{Z}_{21}$ parameters for the two orientations show only minor deviations. More specifically, negligibly small relative deviations in the order of a few percent are observed without bias field. With increasing bias strength and hence decreasing $\mu^{\prime}$, the precise shape of the sense winding plays a more important role. Hence, for a maximal bias current relative deviations up to $20 \%$ are found. To sum up, there is no experimental evidence that indicates a dependence of the measured data on the relative position of the windings.

Description by an empirical analytical relation.-The permeability of fully saturated ferrites is given by the Polder tensor [13]

$$
\overleftrightarrow{\mu}_{d}=\mu_{0}\left(\begin{array}{ccc}
\mu_{\mathrm{diag}} & \mathrm{i} \kappa & 0 \\
-\mathrm{i} \kappa & \mu_{\mathrm{diag}} & 0 \\
0 & 0 & \mu_{z}
\end{array}\right) \quad\left(\text { for } \vec{H}_{0} \| \vec{e}_{z}\right),
$$

with

$$
\begin{aligned}
\mu_{\text {diag }} & =1+\chi, \\
\chi & =\frac{\left(\omega_{0}+\mathrm{i} \omega \alpha\right) \omega_{\mathrm{M}}}{\left(\omega_{0}+\mathrm{i} \omega \alpha\right)^{2}-\omega^{2}}, \\
\kappa & =\frac{-\omega \omega_{\mathrm{M}}}{\left(\omega_{0}+\mathrm{i} \omega \alpha\right)^{2}-\omega^{2}}, \\
\omega_{0} & =-\gamma_{e} \mu_{0} H_{0}, \\
\omega_{\mathrm{M}} & =-\gamma_{e} \mu_{0} M_{\mathrm{sat}}, \\
\mu_{z} & =1 .
\end{aligned}
$$

Therein, $M_{\text {sat }}$ is the saturation magnetization, $\gamma_{e}$ the gyromagnetic ratio, $\alpha$ the loss parameter, and $H_{0}$ the strength of the total effective static magnetic field in $z$ direction, which is composed of the applied dc field $H_{\text {bias }}$ and the crystalline anisotropy field $H_{a}$. A lot of effort has been made to give general analytical expressions for the tensor components $\mu_{\text {diag }}, \kappa$ and $\mu_{z}$ also for partially magnetized states (see, e.g., [14-20]). Since only information about the dynamic response of the Ferroxcube $8 \mathrm{C} 12 \mathrm{~m}$ material in the direction of the static bias field is available from the performed measurements, only terms for the $\mu_{z}$ component of the permeability tensor are investigated in the following. First of all, the partially magnetized state is characterized by the ratio of the magnitude of the magnetization in the $z$ direction to the saturation magnetization $M_{z} / M_{\text {sat }}$. It is worth recalling that, since $M_{z}=$ $B(H) / \mu_{0}-H$, the dependence of $M_{z} / M_{\text {sat }}$ on the bias field strength is directly determined by the $B-H$ curve. As discussed in Sec. IIE, the characteristic function (23) is assumed for the Ferroxcube $8 \mathrm{C} 12 \mathrm{~m}$ ferrite. It is then found empirically that the complex permeability values obtained from the reflection measurements for different magnetization states can be well fitted to the relation

$$
\mu_{z}=1+(\hat{\mu}-1)\left(1-\left(\frac{M_{z}}{M_{\text {sat }}}\right)^{x}\right)
$$

with $H_{0}=H_{\text {bias }}+H_{a}$ and the permeability of the fully demagnetized state [14]

$$
\hat{\mu}=\frac{1}{3}\left(1+2 \sqrt{\mu_{\text {diag }}^{2}-\kappa^{2}}\right)
$$

without claiming any physical basis. A good approximation of the data up to moderate frequencies is observed for setting the free parameters to $\alpha=0.4, H_{a}=195 \mathrm{~A} / \mathrm{m}$ and $x=1.15$. Equation (18) is obtained from the expression derived in [17] from theoretical considerations with two modifications: First, $\mu_{\text {diag }}$ is substituted with $\hat{\mu}$. Second, the exponent $x$ in the spatial averaging, which is denoted with $\langle\ldots\rangle$, in the approximation

$$
1-\left\langle\alpha_{3}^{2}\right\rangle \simeq 1-\left(\frac{M_{z}}{M_{\text {sat }}}\right)^{x}
$$

is left as a free parameter instead of setting it to $x=2$. Therein, $\alpha_{3}$ is the $z$ component of the local effective magnetostatic field normalized to unity (cf. [17]). As discussed above, the ratio $M_{z} / M_{\text {sat }}$ directly depends on the $B-H$ function. Thus, the determined value for the exponent is particularly sensitive to a change of the parameters of the characteristic function.

The obtained analytical relations are included in Fig. 7 as well as in Fig. 9 of the following section together with the measurement data. Note that the analytical expression (19) is only used in this subsection for the comparison with the measurement data. The further derived quantities of the following two subsections only rely on the data from the reflection measurement (cf. Sec. II B).

\section{Reversible permeability as a function of bias magnetic field strength}

The values for the reversible permeability $\mu_{\text {rev }}$ as a function of bias magnetic field strength as obtained from both measurement methods are shown in Fig. 9 and additionally listed in Table IV in the Appendix. The values are evaluated at a frequency of $f=0.5 \mathrm{MHz}$. 


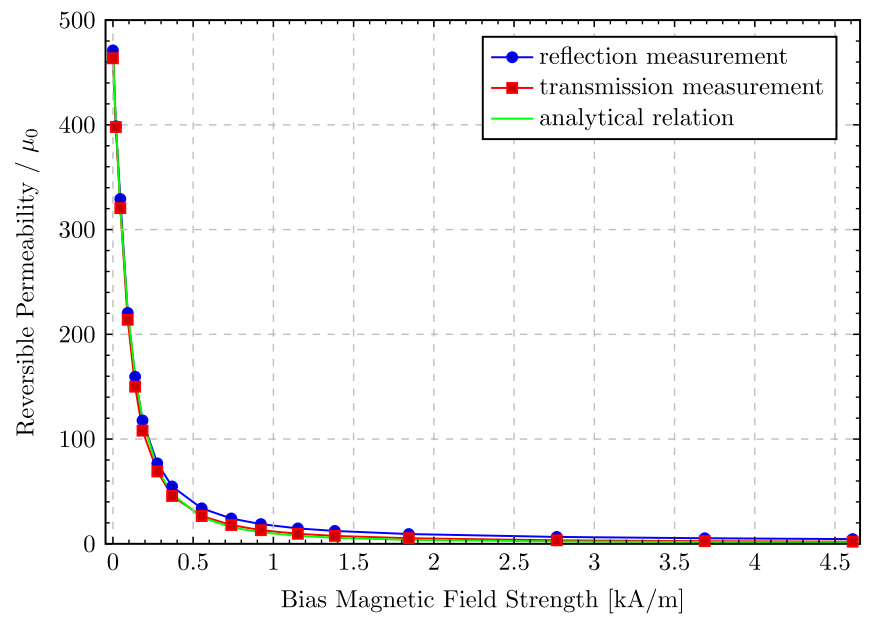

FIG. 9. Real part of the reversible permeability in the low frequency limit, in this study evaluated at a frequency of $f=$ $0.5 \mathrm{MHz}$ as a function of the bias magnetic field strength. Both values as obtained for the analysis of the reflection measurement and of the transmission measurement are shown. The empirically found analytical relation (18) is also plotted for comparison.

\section{Product of $\mu Q f$}

As a further figure of merit, also the $\mu_{\mathrm{s}}^{\prime} Q f$ product evaluated for the reflection measurement data for the series representation is shown in Fig. 10. As expected [21], the $\mu Q f$ curve is shifted to the bottom right with increasing bias field strength.

\section{E. Measurement of the $B$ - $H$ curve of a working cycle with reduced bias range}

In the working cycle of the GSI SIS 18 cavity, the bias current is modified only in the range from zero to its maximum value without changing its polarity. Hence, not the area of the full $B-H$ loop but only a small part near its

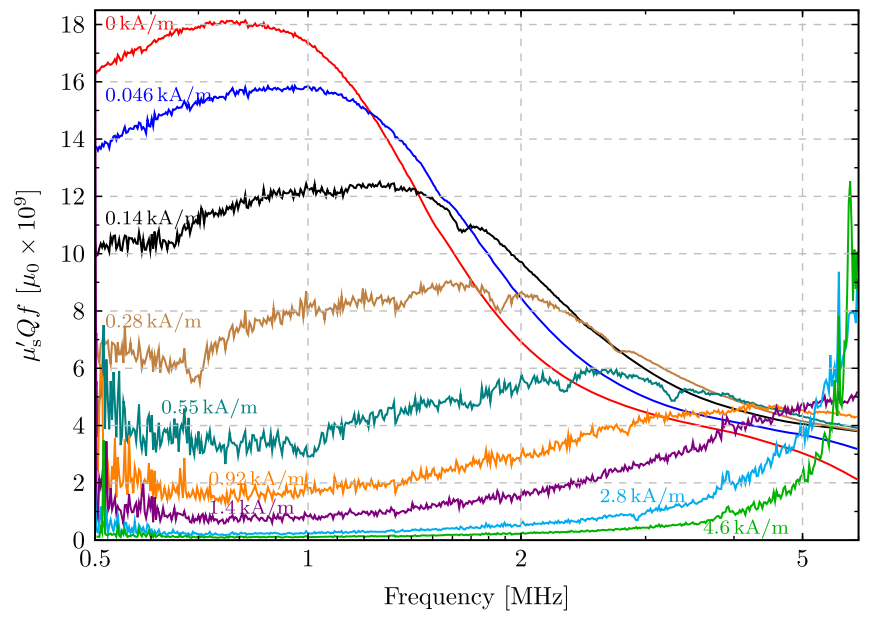

FIG. 10. The $\mu_{\mathrm{s}}^{\prime} Q f$ product for the series representation as obtained from the reflection measurement analysis for different bias field strengths as a function of frequency. upper branch is enclosed. It is the aim of the measurement described in this section to determine the curve surrounding this area by using an approach similar to [4]. The setup which serves for this purpose is as follows. Similar to the previous measurement setup, two full size ferrite ring cores of identical dimensions are biased via $N_{\text {bias }}=24$ crossed (figure-of-eight) current windings. The bias current is again driven by the same dc power supply. Yet, for the $B-H$ loop measurement the output current is controlled by a sinusoidal voltage generated by an Agilent 33522A waveform generator. As a restriction, the maximal effective bias magnetic field was limited to $1.5 \mathrm{kA} / \mathrm{m}$ due to the smaller number of bias windings in this setup. Thus, only a reduced working cycle could be recorded. A voltage monitor output directly proportional to the bias current is connected to the $x$ channel of a LeCroy WaveRunner 44Xi oscilloscope. Taking into account Eq. (8), this output voltage can directly be related to the effective bias magnetic field strength. Moreover, the induced voltage signal from the sense winding with $N_{\text {turns }}=9$ turns is recorded at its $y$ channel and averaged over 50 periods. The induced voltage $U_{\text {ind }}$ is related to the mean magnetic flux density $B$ by

$$
U_{\text {ind }}=N_{\text {turns }} A \frac{\mathrm{d} B}{\mathrm{~d} t},
$$

where $A$ is the area that is penetrated by the magnetic flux. Hence, the magnetic field at the time $t_{n}=t_{n-1}+\Delta t$ can be obtained with numerical integration of the recorded voltage signal as

$$
B\left(t_{n}\right)=\sum_{t_{m}=t_{0}}^{t_{n}} \frac{\Delta t U_{\text {ind }}\left(t_{m}\right)}{N_{\text {turns }}\left(r_{\text {out }}-r_{\text {in }}\right) h_{\text {core }}}+B_{0}
$$

where the inner and outer radius $r_{\text {in }}$ and $r_{\text {out }}$ and the thickness of the ring cores $h_{\text {core }}$ are specified in Table II.

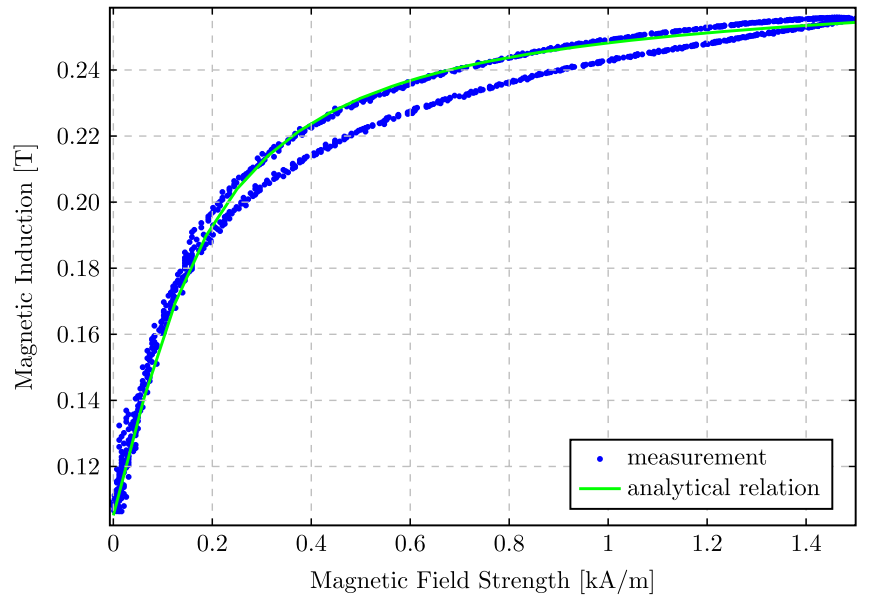

FIG. 11. $B-H$ curve for the working cycle with reduced bias range measured at a frequency of $1 \mathrm{~Hz}$. The solid line represents the characteristic functions (23) with parameters $a=80.4 \mathrm{kA} / \mathrm{m}$, $b=0.0059 \mathrm{~m} / \mathrm{A}$, and $M_{0}=83.7 \mathrm{kA} / \mathrm{m}$. 
The integration constant $B_{0}=B\left(t=t_{0}\right)$ is determined as follows. First of all, a reference value for the saturation magnetization $M_{\text {sat }}$ is required, which is taken as $2.1 \times 10^{5} \mathrm{~A} / \mathrm{m}$. This is the mean of the two values stated in the data sheet [9] for a temperature of $25^{\circ} \mathrm{C}$ and $40^{\circ} \mathrm{C}$. Furthermore, as is observed (cf. Fig. 11), the area enclosed by the $B-H$ curve is reasonably small, which justifies one to neglect hysteresis effects in further considerations. This allows one to choose $B_{0}$ such that the resulting saturation magnetization coincides with $M_{\text {sat }}=2.1 \times 10^{5} \mathrm{~A} / \mathrm{m}$ when fitted to a characteristic function of the form [22]

$$
B(H)=\left[a \arctan (H b)+H+M_{0}\right] \times \mu_{0}
$$

with the free parameters $a, b$, and $M_{0}$. The latter quantity $M_{0}$ may be interpreted as the remanence magnetization. The obtained results for a frequency of $1 \mathrm{~Hz}$ together with Eq. (23) for the parameters $a=80.4 \mathrm{kA} / \mathrm{m}, b=$ $0.0059 \mathrm{~m} / \mathrm{A}$, and $M_{0}=83.7 \mathrm{kA} / \mathrm{m}$ are shown in Fig. 11. Moreover, the $B-H$ loop measurements were repeated at the frequencies 5 and $10 \mathrm{~Hz}$. The obtained curves for all three frequencies are fully in accordance with each other, albeit some slight distortions are observed for the measurement at $10 \mathrm{~Hz}$.

\section{SUMMARY AND CONCLUSION}

The measurement of the magnetic material characteristics of the Ferroxcube $8 \mathrm{C} 12 \mathrm{~m}$ ferrite in the parameter range where the GSI SIS 18 cavity is operated has been discussed. Among these characteristics are the complex permeability as a function of frequency and bias magnetic field strength as well as the $B-H$ curve. The former is determined in two different approaches, one in a reflection and one in a transmission setup. The obtained permeability values for low rf levels are fully compatible with each other within their estimated error margins up to moderate frequencies. Beyond that, the transmission approach is not applicable anymore due to $L C$ resonances. Moreover, an analytical relation for the real and imaginary part of the permeability is found empirically, which properly describes the dependence on the bias magnetic field strength and the frequency in the whole investigated frequency range.

The obtained material data were already successfully used in numerical eigenmode simulations [23] of the GSI SIS 18 ferrite cavity. Those simulations rely on accurate measurement data since they fully take into account the dependence of the complex permeability on the bias magnetic field strength as well as on the frequency. The numerical computations are, however, beyond the scope of this report and will be presented separately.

\section{ACKNOWLEDGMENTS}

This work is supported by the GSI Helmholtzzentrum für Schwerionenforschung GmbH, Darmstadt, Germany.
TABLE IV. Real part of the reversible permeability $\mu_{\text {rev }}$ in the low frequency limit, in this study evaluated at a frequency of $f=0.5 \mathrm{MHz}$. Both values as obtained for the analysis of the reflection measurement and of the transmission measurement are given. The values from the empirically found analytical relation (18) are also listed for comparison.

\begin{tabular}{lccc}
\hline \hline & \multicolumn{3}{c}{$\mu_{\text {rev }} / \mu_{0}$} \\
\cline { 2 - 4 }$H_{\text {bias }}[\mathrm{kA}]$ & $\begin{array}{c}\text { reflection } \\
\text { measurement }\end{array}$ & $\begin{array}{c}\text { transmission } \\
\text { measurement }\end{array}$ & $\begin{array}{c}\text { analytical } \\
\text { relation }\end{array}$ \\
\hline 0 & 471 & 464 & 470 \\
0.02 & 399 & 398 & 402 \\
0.05 & 329 & 320 & 320 \\
0.09 & 220 & 214 & 223 \\
0.14 & 160 & 150 & 160 \\
0.18 & 118 & 108 & 119 \\
0.28 & 77 & 69 & 72 \\
0.37 & 55 & 46 & 47 \\
0.55 & 34 & 27 & 25 \\
0.74 & 24 & 18 & 16 \\
0.92 & 19 & 13 & 11 \\
1.15 & 15 & 9.6 & 7.7 \\
1.38 & 12 & 7.6 & 5.7 \\
1.84 & 9.3 & 5.3 & 3.8 \\
2.77 & 6.6 & 3.3 & 2.3 \\
3.69 & 5.3 & 2.5 & 1.7 \\
4.61 & 4.4 & 2.0 & 1.5 \\
\hline \hline
\end{tabular}

\section{APPENDIX: VALUES FOR THE REVERSIBLE PERMEABILITY IN TABULAR FORM}

The values for the reversible permeability $\mu_{\text {rev }}$ as a function of bias magnetic field strength $H_{\text {bias }}$ as obtained from the reflection and the transmission measurement method, which are also shown in Fig. 9, are listed in Table IV.

[1] Q. Yu, T. Holmes, and K. Naishadham, RF equivalent circuit modeling of ferrite-core inductors and characterization of core materials, IEEE Transactions on Electromagnetic Compatibility 44, 258 (2002).

[2] Agilent Solutions for Measuring Permittivity and Permeability with LCR Meters and Impedance Analyzers, application Note 1369-1, 2008.

[3] J. Shenhui and J. Quanxing, An alternative method to determine the initial permeability of ferrite core using network analyzer, IEEE Transactions on Electromagnetic Compatibility 47, 651 (2005).

[4] V. J. Thottuvelil, T. G. Wilson, and H. A. Owen Jr., Highfrequency measurement techniques for magnetic cores, IEEE Trans. Power Electron. 5, 41 (1990).

[5] F. Fiorillo, Measurements of magnetic materials, Metrologia 47, S114 (2010). 
[6] C. Vollinger, F. Caspers, and E. Jensen, The effect of 2-directional magnetic biasing used for tuning of a ferriteloaded re-entrant cavity, IEEE Trans. Nucl. Sci. 60, 2170 (2013).

[7] Delta Elektronika Data sheet SM3000-series, 2012 [http:// www.delta-elektronika.nl/upload/dts_sm3000.pdf].

[8] Rohde \& Schwarz GmbH \& Co. KG, ZNB Vector Network Analyzers User Manual, 2012 [http://www.rohde-schwarz .com].

[9] Ferroxcube, Soft Ferrites and Accessories, 2009 [http:// ferroxcube.home.pl/appl/info/HB2009.htm].

[10] TDK Corporation, EMC Technology—Guide Book For EMC, 2011 [http://www.tdk.co.jp/emc_guide_e/].

[11] Rohde \& Schwarz GmbH \& Co. KG, ZNB Vector Network Analyzers Specifications, 2013, version 06.00 [http://www .rohde-schwarz.com].

[12] D. M. Pozar, Microwave Engineering, 3rd ed. (John Wiley \& Sons, New Jersey, 2005).

[13] D. Polder, On the theory of ferromagnetic resonance, Philos. Mag. 40, 99 (1949).

[14] E. Schlömann, Microwave behavior of partially magnetized ferrites, J. Appl. Phys. 41, 204 (1970).

[15] G. T. Rado, Theory of the microwave permeability tensor and Faraday effect in nonsaturated ferromagnetic materials, Phys. Rev. 89, 529 (1953).
[16] J. J. Green and F. Sandy, Microwave characterization of partially magnetized ferrites, IEEE Trans. Micro. Theory Techn. 22, 641 (1974).

[17] M. Igarashi and Y. Naito, Parallel component $\mu_{z}$ of partially magnetized microwave ferrites, IEEE Trans. Micro. Theory Techn. 29, 568 (1981).

[18] J. P. Bouchaud and P. G. Zérah, Spontaneous Resonances and Universal Behavior in Ferrimagnets: EffectiveMedium Theory, Phys. Rev. Lett. 63, 1000 (1989).

[19] P. Gelin, New consistent model for ferrite permeability tensor with arbitrary magnetization state, IEEE Trans. Micro. Theory Techn. 45, 1185 (1997).

[20] P. Gelin and P. Queffelec, Generalized permeability tensor model: Application to barium hexaferrite in a remanent state for self-biased circulators, IEEE Trans. Magn. 44, 24 (2008).

[21] F. G. Brockman, H. van der Heide, and M. W. Louwerse, Ferroxcube für Protonensynchrotrons, Philips Technische Rundschau (1969).

[22] A. Bergqvist, Magnetic vector hysteresis model with dry friction-like pinning, Physica B (Amsterdam) 233, 342 (1997).

[23] K. Klopfer, W. Ackermann, and T. Weiland, Computation of complex eigenmodes for resonators filled with gyrotropic materials, IEEE Trans. Magnet. (to be published), doi: 